\title{
Pushing size to the limit: extreme DNA
}

Double-wide pyrimidine bases can assemble into helical, sequence-specific DNA complexes.

Though it is one of the first things any biology student learns about genetics, it is always amazing to stop and consider that the same four lowly DNA nucleotides are the foundation for the enormous diversity of life. Though the genetic code evolved as we know it because it is so stable and efficient, several creative research groups have begun to show that these four bases are not the only molecules capable of storing genetic information, nor is there a hard and fast limit to the type of information that can be replicated.

The laboratory of Eric Kool at Stanford University is one of the leading groups in this innovative field. Toward their goal of creating an artificial genetic system, Kool and his colleagues recently began investigating the question of whether larger DNA bases could replace their natural counterparts and still assemble into sequence-specific helices. Encouraged by results where they widened DNA bases by $2.4 \AA$ (Lu et al., 2004), Kool and postdoctoral student Alex Lee demonstrated in a recent report that DNA strands containing 'double-wide' pyrimidine bases, widened by $4.8 \AA$, can self-assemble into relatively stable helices (Lee \& Kool, 2006).

Lee and Kool synthesized double-wide cytidine (yyC) and thymidine (yyT) by inserting a 4.8 A naphthalene moiety between the sugar and the nucleobase. The insertion was situated just so that neither the sugar backbone nor the hydrogen bonding capabilities of the bases were substantially altered. Notably, these double-wide bases were inherently fluorescent, which could facilitate their application in various imaging experiments.

To test whether yyC and yyT could be incorporated into DNA helices, Lee and Kool prepared several DNA strands containing a mixture of yyC, yyT, guanine and adenine, or containing only yyC and yy to complement a strand of only guanine and adenine. They used several methods to test helicity, including UV-monitored thermal melting, circular dichroism and fluorescence resonance energy transfer. When placed in natural DNA, yyC and yyT were moderately destabilizing to the helix, but when the strands contained all
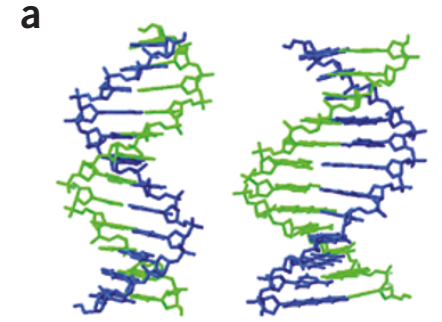

b
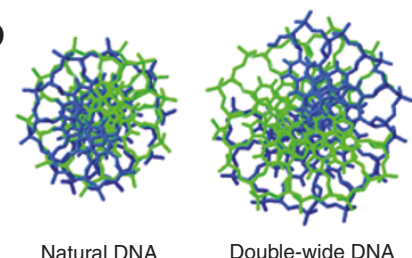

Double-wide DNA

Figure 1 | Molecular models comparing the relative sizes of natural and double-wide DNA composed of $y \mathrm{y} T \bullet A$ and $\mathrm{yy} C \bullet \mathcal{C}$ pairs. $(\mathbf{a}, \mathbf{b})$ Side views (a) and end views (b). Reprinted with permission from Journal of the American Chemical Society. Copyright 2006, American Chemical Society.

doubly widened pairs, they did form helices (Fig. 1). "It is a little surprising that [helices form] at all," says Kool. "We think that the exceptionally strong stacking of the doublewide bases pays for any cost of adjusting the sugar-phosphate backbone to the large size."

Several studies have shown that molecular geometry and packing are the most important considerations when creating new DNA base pairs. "The most important issue in DNA helix assembly is not the size of the pairs - it's the fact that all the pairs have the same geometry, leading to a regular conformation in the polymer," Kool explains. The larger base size could be troublesome in the next step toward creating a replicating system, as it will be challenging to find or design a polymerase that will accept widened DNA, but making 'extreme DNA' is certainly a unique and potentially useful approach toward designing a new genetic system.

\section{Allison Doerr}

\section{RESEARCH PAPERS}

Lu, H. et al. yDNA: a new geometry for size-expanded base pairs. Angew. Chem. Int. Ed. 43, 5834-5836 (2004).

Lee, A.H.F. \& Kool, E.T. Exploring the limits of DNA size: naphtho-homologated DNA bases and pairs. $J$. Am. Chem. Soc. 128, 9219-9230 (2006). 